Pacific Journal of Mathematics

HEIGHT ESTIMATES FOR EXTERIOR PROBLEMS OF 


\title{
HEIGHT ESTIMATES FOR EXTERIOR PROBLEMS OF CAPILLARITY TYPE
}

\section{BRUCE TURKINGTON}

\begin{abstract}
This work concerns boundary value problems for a class of nonlinear equations modeled on the physical equations for a capillary free surface in a gravitational field. The results consist principally of estimates for the height of a solution in an exterior domain. Structure conditions reflecting the nonlinearity of the mean curvature operator are imposed on a class of symmetric variational operators in terms of the Legendre transform of the variational integrand. Estimates are found for the boundary height of a rotationally symmetric solution in the exterior of a ball of radius $R$. These estimates, which are valid for any $R$, are shown to be asymptotically exact as $R$ tends to zero or infinity. This provides a proof of the asymptotic behavior of the boundary height which previously has been derived by a formal perturbation method. An asymptotic characterization of the solution in a neighborhood of the boundary is also given. For a general domain estimates are obtained from a maximum principle due to Finn in which the symmetric solutions serve as comparison functions.
\end{abstract}

1. Preliminaries. We begin by formulating the "standard" capillarity problem on an exterior domain $n$ dimensional space; the physical case is $n=2$. Let $\Omega \subset \boldsymbol{R}^{n}$ be an exterior domain whose boundary, $\Sigma$, is a compact $C^{1}$ hypersurface. When the generalized (vertical) cylinder $\Sigma \times \boldsymbol{R}$ is immersed in an infinite reservoir of fluid, the action of capillarity gives rise to a free surface of static equilibrium in the outside of the cylinder. Let the height of this capillary free surface, assumed nonparametric over $\Omega$, be given by the scalar function $u(x), x=\left(x_{1}, \cdots, x_{n}\right) \in \Omega$. Physical principles assert that the equilibrium free surface minimizes the functional

$$
E[u]=\sigma \int_{\Omega}\left(\sqrt{1}+|\nabla u|^{2}-1\right) d x+\frac{1}{2} \rho g \int_{\Omega} u^{2} d x-\sigma \beta \int_{\Sigma} u d S
$$

$d x$ is $n$ dimensional measure on $\Omega$ and $d S$ is $(n-1)$ dimensional measure on $\Sigma$. The physical constants appearing in (1.1) are: $\sigma$, the surface tension; $\rho$, the difference of densities across the free surface; and, $g$, the gravitational acceleration. The dimensionless constant $\beta$ is a characteristic of the materials interfacing at the boundary; $\beta$ satisfies $|\beta| \leqq 1$ and $\gamma=\cos ^{-1} \beta(0 \leqq \gamma \leqq \pi)$ is known as the contact angle. The equilibrium condition $\delta E[u]=0$ yields the Euler equations 


$$
\begin{aligned}
& \nabla \cdot\left\{\left(1+|\nabla u|^{2}\right)^{-1 / 2} \nabla u\right\}=\kappa u \text { in } \quad \Omega \\
& \nu \cdot\left\{\left(1+|\nabla u|^{2}\right)^{-1 / 2} \nabla u\right\}=\beta \quad \text { on } \quad \Sigma
\end{aligned}
$$

where $\kappa=\rho g / \sigma$ is the capillarity constant and $\nu$ is the outer unit normal on $\Sigma$. We note that in (1.2)

$$
M u=\nabla \cdot\left\{\left(1+|\nabla u|^{2}\right)^{-1 / 2} \nabla u\right\}
$$

is the mean curvature operator. When considering an exterior domain $\Omega$ it is natural to require that

$$
\lim _{x \rightarrow \infty} u(x)=0 \text {; }
$$

in fact, it can be shown that this property is possessed by any solution of equation (1.2) defined in a neighborhood of infinity.

The questions of existence and regularity for solutions of $(1.2,1.3)$ under certain restrictions on the exterior domain $\Omega$ and the boundary data $\beta$ have been dealt with by Gerthardt $[5,6]$.

We consider a class of symmetric variational equations generalizing the capillarity equations $(1.2,1.3)$. While the analysis to follow is carried out using methods appropriate to the general case, the theorems yield new results when specialized to the case of capillary free surfaces.

Let $F(s) \in C^{2}[0, \infty)$ and $G(u) \in C^{2}(-\infty, \infty)$ be given satisfying

$$
\begin{gathered}
F_{s}(0)=0 \text { and } F_{s s}(s)>0 \text { for all } s \in[0, \infty), \\
G(0)=G_{u}(0)=0 \text { and } G_{u u}(u)>0 \text { for all } u \in(-\infty, \infty) .
\end{gathered}
$$

For convenience we write $F(p)$ when considering $F(s)$ as a function of $p=\left(p_{1}, \cdots, p_{n}\right), s=|p|$.

We consider the boundary value problem ${ }^{1)}$

$$
\begin{gathered}
\frac{\partial}{\partial x_{i}} F_{p_{i}}(\nabla u)=G_{u}(u) \text { in } \Omega \\
\nu_{i} F_{p_{i}}(\nabla u)=\varphi_{u}(x, u) \text { on } \Sigma \text {; }
\end{gathered}
$$

namely, the Euler equations for the functional

$$
J[u]=\int_{\Omega}\{F(|\nabla u|)+G(u)\} d x-\int_{\Sigma} \varphi(x, u) d S .
$$

Here, $\varphi(x, u) \in C^{2}(\Sigma \times \boldsymbol{R})$ is prescribed boundary data and may have to satisfy certain conditions depending on $F(s)$. Strictly speaking we must seek solutions $u(x)$ of $(1.8,1.9)$ in the class $C^{2}(\Omega) \cap C^{1}(\bar{\Omega})$; however, it is of interest to consider solutions $u(x)$ which are not

\footnotetext{
1 The summation convention is used on repeated indices.
} 
in $C^{1}(\bar{\Omega})$. For $u(x) \in C^{2}(\Omega) \cap C^{0}(\bar{\Omega})$ we may replace (1.9) by the condition

$$
\lim _{x \rightarrow x_{0} x \in 2} \nu_{\imath} F_{p_{\imath}}(\nabla u)=\left.\varphi_{u}(x, u)\right|_{x=x_{0}} \text { for all } x_{0} \in \Sigma
$$

provided this limit exists ( $\nu$ is extended continuously to a neighborhood of $\Sigma$ ).

It is evident from (1.6) that the quasilinear operator

$$
N u=\frac{\partial}{\partial x_{i}} F_{p_{i}}(\nabla u)
$$

appearing in (1.8) is elliptic. In $\S 2$ we impose structure conditions on $N u$ making it the appropriate generalization (for our purposes) of the mean curvature operator $M u$. Under these conditions any solution of $(1.8,1.9)$ will satisfy the natural requirement $(1.5)$.

The following variant of a maximum principle due to Concus and Finn $[1,2]$ is fundamental to our discussion.

THEOREM 1.1. Suppose $\varphi_{u x}(x, u) \leqq 0$ for all $(x, u) \in \Sigma \times \boldsymbol{R}$. Let $u(x), v(x) \in C^{2}(\Omega) \cap C^{0}(\bar{\Omega})$ satisfy

$$
\begin{array}{ll}
\frac{\partial}{\partial x_{i}} F_{p_{i}}(\nabla u) \leqq G_{u}(u) & x \in \Omega \\
\lim _{x \rightarrow x_{0}, x \in \Omega} \inf _{i} F_{p_{i}}(\nabla u) \geqq\left.\varphi_{u}(x, u)\right|_{x=x_{0}} & x_{0} \in \Sigma \\
\frac{\partial}{\partial x_{i}} F_{p_{i}}(\nabla v) \geqq G_{u}(v) & x \in \Omega \\
\lim _{x \rightarrow x_{0}, x \in !} \sup _{2} \nu_{p_{i}}(\nabla v) \leqq\left.\varphi_{u}(x, v)\right|_{x=x_{0}} & x_{0} \in \Sigma
\end{array}
$$

and $\lim _{x \rightarrow \infty} u(x)=\lim _{x \rightarrow \infty} v(x)=0$. Then $u(x) \geqq v(x), x \in \Omega$.

We say $u(x)$ is a supersolution and $v(x)$ is a subsolution of problem $(1.8,1.9)$.

Proof. The proof given in Concus and Finn $[1,2]$ for a bounded domain $\Omega$ and with $F(s)=\sqrt{1+s^{2}}$ is easily adapted to yield this theorem.

Throughout the remainder of the paper we shall assume that the hypothesis

$$
\varphi_{u u}(x, u) \leqq 0 \text { for all }(x, u) \in \Sigma \times \boldsymbol{R}
$$

is fulfilled by the prescribed boundary data. For capillary free surfaces this means that we may consider cylinders $\Sigma \times \boldsymbol{R}$ composed of inhomogeneous material provided that the contact angle $\gamma(x, u)$ is nondecreasing in $u$.

Since much of the analysis to follow will concern rotationally 
symmetric solutions $u(r), r=|x|$, on the domain $\widetilde{B}_{R}=\{x:|x|>R\}$ (or the limiting case when $R \rightarrow \infty$ and $u$ is a one dimensional solution on a half-space), we extend the definition of $F(s)$ to $s \in(-\infty, \infty)$ in order to make convenient the application of $N$ to $u(r)$. If $F_{s}(s)$ is extended as an odd function on $(-\infty, \infty)$ then $F(s)$ becomes an even $C^{2}$ function on $(-\infty, \infty)$ and, furthermore, we have

$$
F_{p_{i}}(\nabla u)=F_{s}\left(u_{r}\right) \frac{x_{i}}{r}
$$

when $u_{r}$ takes either sign. When considering rotationally symmetric solutions we must restrict the boundary data to be of the form

$$
\varphi(x, u)=\beta u, \quad \beta=\text { constant }
$$

as in the capillarity problem with constant contact angle. The boundary value problem $(1.8,1.9)$ for $u(r)$ on $\widetilde{B}_{R}$ is given by the ordinary differential equations

$$
\begin{gathered}
\frac{1}{r^{n-1}} \frac{d}{d r}\left(r^{n-1} F_{s}\left(u_{r}\right)\right)=G_{u}(u) \quad r>R \\
-F_{s}\left(u_{r}\right)=\beta \quad r=R .
\end{gathered}
$$

The following theorem, derived from the preceding maximum principle, allows rotationally symmetric solutions to be used as comparison functions.

Theorem 1.2. Suppose $\Omega \subset \widetilde{B}_{R}$ for some $R>0$. Let $u(x)$ be a solution of $(1.8,1.9)$ in $\Omega$ and let $v(r)$ be a solution of $(1.17,1.18)$ in $\widetilde{B}_{R}$ with $v_{r}<0$ and $v_{r r}>0$ for $r>R$. If $\varphi_{u}(x, u(x)) \geqq \beta$ for all $x \in \Sigma$, then $u \geqq v$ in $\Omega$.

Proof. By Theorem 1.1 it suffices to show that $\nu_{i} F_{p_{i}}(\nabla u) \geqq$ $\nu_{i} F_{p_{i}}(\nabla v)$ on $\Sigma$. Let $\xi_{i}=v_{x_{i}}|| \nabla v \mid$. For $x \in \Sigma, \nu_{i} F_{p_{i}}(\nabla v) \leqq \xi_{i} F_{p_{i}}(\nabla v)=$ $F_{s}(|\nabla v|)=-F_{s}\left(v_{r}(|x|)\right)$. Using (1.6) and $v_{r r}>0$ we obtain

$$
-F_{s}\left(v_{r}(|x|)\right)<-F_{s}\left(v_{r}(R)\right),
$$

hence $\nu_{i} F_{p_{i}}(\nabla v)<-F_{s}\left(v_{r}(R)\right)=\beta \leqq \varphi_{u}(x, u(x))=\nu_{i} F_{p_{i}}(\nabla u)$ as required.

As is evident from the proof, the theorem also holds when $v_{r}>0$ and $v_{r r}<0$. For a given domain $\Omega$ the preceding theorem holds, of course, for any ball contained in the complement of $\Omega$.

This theorem, allowing the comparison of two capillary free surfaces defined on two different domains, is due to Finn [4]. As is pointed out in [4], the result is false for two arbitrary domains, one containing the other. A complementary result to Theorem 1.2 
is the following corollary of Theorem 1.1: If $\Omega \supset \widetilde{B}_{R}$ for some $R>0$ and $u(x)$ is a solution of $(1.8,1.9)$ in $\Omega$ and $w(r)$ is a solution of (1.17) in $\widetilde{B}_{R}$ with $w_{r}(R)=-\infty$, then $u \leqq w$ in $\widetilde{B}_{R}$.

In view of the above results we focus our attention on height estimates for rotationally symmetric solutions on $\widetilde{B}_{R}$. In $\S 2$ we find structure conditions on the operator $N u$ which are necessary and sufficient for the existence of solutions $u=u(r ; R, \beta)$ of $(1.17,1.18)$ for all appropriate $\beta$. The boundary height $u(R ; R, \beta)$ is characterized asymptotically as $R \rightarrow 0$ and as $R \rightarrow \infty$ in $\S 3$. The results of $\S 3$ are applied in $\S 4$ to give asymptotic estimates on the solution $u(r$; $R, \beta)$ in a neighborhood of the boundary as $R \rightarrow 0$.

2. Structure conditions. By structure conditions on the operator $N u$ defined by (1.11) we mean conditions on the behavior of $F(s)$ as $s \rightarrow \infty$; these we impose in terms of the Legendre transform of $F(s)$.

The Legendre transform $(\sigma, \Phi(\sigma))$ of $(s, F(s))$ is defined to be

$$
\sigma=F_{s}, \Phi=-s \sigma+F .
$$

We note that $\Phi$ may be considered as a function of the new independent variable $\sigma$ because of the Legendre condition (1.6). Clearly, $\sigma$ is an odd $C^{1}$ function of $s$ and $\Phi$ is an even $C^{1}$ function of $\sigma$. The transformation possesses the involutive property:

$$
\Phi_{\sigma}=-s .
$$

Our requirement is that both $\sigma$ and $\Phi$ be bounded as $s \rightarrow \infty$; in fact, it is not hard to show that the boundedness of $\Phi$ implies the boundedness of $\sigma$.

Since $\sigma$ is strictly increasing in $s, \lim _{s \rightarrow \infty} \sigma$ exists. Multiplying equations $(1.8,1.9)$ by a positive constant we may assume that

$$
\lim _{s \rightarrow \infty} \sigma=1 \text {. }
$$

This normalization constrains the boundary data in (1.9) since

$$
\left|\varphi_{u}(x, u)\right| \leqq\left\{\sum_{1}^{n} F_{p_{i}}(\nabla u)^{2}\right\}^{1 / 2}=F_{s}(|\nabla u|) \leqq 1 .
$$

Henceforth we assume that the boundary data is prescribed so that

$$
\left|\varphi_{u}(x, u)\right| \leqq 1 \text { for all }(x, u) \in \Sigma \times \boldsymbol{R} .
$$

In particular, in (1.18) we have $|\beta| \leqq 1$. Also, since $\Phi$ is strictly decreasing in $\sigma, \lim _{\sigma \rightarrow 1} \Phi$ exists. As equations $(1.8,1.9)$ are unchanged when $\Phi$ and $F$ are replaced by $\Phi+C$ and $F+C$ we may assume, after an appropriate choice of $C$, the normalized requirement 


$$
\lim _{\sigma \rightarrow 1} \Phi=0 \text {. }
$$

The two (normalized) structure conditions $(2.3,2.5)$ on the operator $\mathrm{Nu}$ will be assumed throughout. For the capillarity problem, where $N u$ is the mean curvature operator, $F(s)=\sqrt{1+s^{2}}$ and hence

$$
\sigma=\frac{s}{\sqrt{1+s^{2}}}, \quad \Phi=\sqrt{1-\sigma^{2}}
$$

clearly $(2.3,2.5)$ are satisfied in this case.

The rotationally symmetric solutions on $\widetilde{B}_{R}$ are supplied by the following theorem.

THEOREM 2.1. There exists a unique solution $u=u(r ; R, \beta)$ of (1.17, 1.18) for every $0<R<\infty$ and $0 \leqq \beta \leqq 1$. For $0 \leqq \beta<1$, $u \in C^{2}(R, \infty) \cap C^{1}[R, \infty)$; for $\beta=1, u \in C^{2}(R, \infty) \cap C^{0}[R, \infty)$. If $\beta=0$, then $u \equiv 0$; if $0<\beta \leqq 1$, then $u>0, u_{r}<0, u_{r r}>0, \lim _{r \rightarrow \infty} u=\lim _{r \rightarrow \infty} u_{r}=0$.

This result is a direct generalization of the work of the work of Johnson and Perko [9] for two dimensional capillary free surfaces. Uniqueness is immediate from the maximum principle, Theorem 1.1. A detailed proof of existence is contained in Perko [10]; under conditions $(1.6,1.7)$, our structure conditions are equivalent to the hypotheses of the latter paper.

In the above theorem it suffices to consider $0 \leqq \beta \leqq 1$ since for $-1 \leqq \beta \leqq 0$ we may take the solution $u(r)=-\widetilde{u}(r)$ where $\widetilde{u}(r)$ solves $(1.17,1.18)$ with $G_{u}(u)$ replaced by $-G_{u}(-\tilde{u})$ and $\beta$ replaced by $-\beta$.

The operator $N u$ when applied to $u=u(r ; R, \beta)$ may be expressed in a form fundamental to the subsequent analysis. From (2.2) it follows that

$$
\begin{aligned}
\frac{d}{d r} F_{s}\left(u_{r}\right) & =u_{r} \frac{d}{d u} F_{s}\left(u_{r}\right) \\
& =-\Phi_{\sigma}\left(F_{s}\left(u_{r}\right)\right) \frac{d}{d u} F_{s}\left(u_{r}\right) \\
& =-\frac{d}{d u} \Phi\left(F_{s}\left(u_{r}\right)\right)
\end{aligned}
$$

Therefore we have

$$
N u=-\frac{d}{d u} \Phi\left(F_{s}\left(u_{r}\right)\right)+\frac{n-1}{r} F_{s}\left(u_{r}\right) .
$$

We remark that the structure conditions imposed on $\sigma$ and $\Phi$ are, in fact, necessary for the existence of a solution $u=u(r ; R, 1)$ with $u \in C^{2}(R, \infty) \cap C^{0}[R, \infty)$. Let $\hat{R} \in(R, \infty)$ be fixed. Integrating 
(1.17) in $r$ and then, using (2.8), in $u$ we obtain respectively

$$
\begin{gathered}
\hat{R}^{n-1} F_{s}\left(u_{r}(\hat{R})\right)-R^{n-1} F_{s}\left(u_{r}(R)\right)=\int_{R}^{\hat{R}} r^{n-1} G_{u}(u(r)) d r \\
\Phi\left(F_{s}\left(u_{r}(\hat{R})\right)\right)-\Phi\left(F_{s}\left(u_{r}(R)\right)\right)+\int_{u(\hat{R})}^{u(R)} \frac{n-1}{r} F_{s}\left(u_{r}\right) d u=G(u(R))-G(u(\hat{R})) .
\end{gathered}
$$

Since $\sup _{r \in(R, \hat{R})} u(r)=u(R)<\infty$ and $\left|u_{r}(\hat{R})\right|<\infty$, it follows that $\left|F_{s}\left(u_{r}(R)\right)\right|,\left|\Phi\left(F_{s}\left(u_{r}(R)\right)\right)\right|<\infty$. But $u_{r}(R)=-\infty$ since $\beta=1$; hence $\sigma=F_{s}$ and $\Phi\left(F_{s}\right)$ are bounded as $s \rightarrow \infty$.

The one-dimensional solutions $u\left(x_{1}\right)$ on the half-space $H_{1}=$ $\left\{x \in \boldsymbol{R}^{n}: x_{1}>0\right\}$ can be considered as the limiting form of the solutions $u(r ; R, \beta)$ as $R \rightarrow \infty$. To simplify notation we write $x=x_{1}$. The equations for $u(x)$ are

$$
\begin{gathered}
\frac{d}{d x} F_{s}\left(u_{x}\right)=G_{u}(u) \quad x>0 \\
-F_{s}\left(u_{x}\right)=\beta \quad x=0 .
\end{gathered}
$$

THEOREM 2.2. There exists a unique solution $u=u(x ; \infty, \beta)$ of $(2.9,2.10)$ for every $0 \leqq \beta \leqq 1$. For $0 \leqq \beta<1, u \in C^{2}(0, \infty) \cap C^{1}[0, \infty)$; for $\beta=1, u \in C^{2}(0, \infty) \cap C^{0}[0, \infty)$. If $\beta=0$, then $u \equiv 0$; if $0<\beta \leqq 1$, then $u>0, u_{x}<0, u_{x x}>0, \lim _{x \rightarrow \infty} u=\lim _{x \rightarrow \infty} u_{x}=0$.

Proof. We may assume $\beta \neq 0$. Let $u_{\infty, \beta}$ be determined by the relation

$$
G\left(u_{\infty, \beta}\right)=\Phi(0)-\Phi(-\beta) .
$$

An explicit formula for the inverse of the solution is given by

$$
x(u)=\int_{u}^{u_{\infty}, \beta} \frac{d u^{\prime}}{F_{s}^{-1}\left(\Phi^{-1}\left(\Phi(0)-G\left(u^{\prime}\right)\right)\right)}, 0<u \leqq u_{\infty, \beta},
$$

where $\Phi^{-1}$ is the positive branch. To derive this formula we observe that by (2.7) (with $r$ replaced by $x$ ) equation (2.9) admits the first integral

$$
\Phi\left(F_{s}\left(u_{x}\right)\right)+G(u)=\Phi(0), \quad x>0 .
$$

The stated properties of the solutiou are now easily verified.

3. Boundary height estimates. In this section we find estimates for the boundary height $u(R ; R, \beta)$ of the solutions of Theorem 2.1. These results allow us to characterize $u(R ; R, \beta)$ asymptotically as $R \rightarrow 0$ and as $R \rightarrow \infty$. The estimation is achieved by comparing certain expressions in the solution $u(r)$ of the given equation $N u=$ 
$G_{u}$ with corresponding expressions in $z(r)$ where $z(r)$ is the solution of the homogeneous equation $N z=0$.

Let $z=z(r ; R, \beta)$ be the solution of

$$
\begin{gathered}
N z=0 \quad r>R \\
z=0,-F_{s}\left(z_{r}\right)=\beta \quad r=R .
\end{gathered}
$$

An integration yields the solution explicitly:

$$
z(r ; R, \beta)=\int_{R}^{r} F_{s}^{-1}\left(-\beta(R / \rho)^{n-1}\right) d \rho .
$$

The solution satisfies $z<0, z_{r}<0, z_{r r}>0$ on $r>R$ for $0<\beta \leqq 1$. When $\beta=1$ the integrand of (3.3) becomes singular at $r=R$; its integrability can be verified using conditions $(2.3,2.5)$. We let $r(z ; R, \beta)$ denote the inverse function of $z(r ; R, \beta)$.

THEOREM 3.1. Let $u=u(r ; R, \beta)$ be the solution of Theorem 2.1 with $0<\beta \leqq 1$ and let $u_{R, \beta}=u(R ; R, \beta)$. Then there holds for all $0<R<\infty$

$$
T(R ; \beta)<u_{R, \beta}<u_{\infty, \beta}=G^{-1}(\Phi(0)-\Phi(-\beta))
$$

where $T(R ; \beta)$ is a positive, increasing, $C^{1}$ function of $R$ defined implicitly by

$$
S(T / R)=G(T)
$$

with

$$
S(w)=(n-1) \beta \int_{z(\infty ; 1, \beta)}^{-w} r(z ; 1, \beta)^{-n} d z .
$$

Proof. Using (2.8) we express equation (1.17) in the form

$$
-\frac{d}{d u} \Phi\left(F_{s}\left(u_{r}\right)\right)+\frac{n-1}{r} F_{s}\left(u_{r}\right)=\frac{d}{d u} G(u),
$$

from which we conclude

$$
\Phi(0)-\Phi(-\beta)+\int_{0}^{u_{R, \beta}} \frac{n-1}{r} F_{s}\left(u_{r}\right) d u=G\left(u_{R, \beta}\right) .
$$

Since the integral appearing in (3.8) is negative we immediately obtain the upper bound in (3.4). To find the lower bound we must estimate this integral. Integrating (3.1) in $z$ we are lead to

$$
\Phi(0)-\Phi(-\beta)+\int_{z(\infty ; R, \beta)}^{0} \frac{n-1}{r} F_{s}\left(z_{r}\right) d z=0
$$


where

$$
z(\infty ; R, \beta)= \begin{cases}-\infty & n=2 \\ \text { finite } & n>2\end{cases}
$$

We now claim the following:

$$
-\int_{0}^{u_{R, \beta}} \frac{n-1}{r} F_{s}\left(u_{r}\right) d u<-\int_{-u_{R}, \beta}^{0} \frac{n-1}{r} F_{s}\left(z_{r}\right) d z .
$$

It is convenient to set $y(r)=z(r)+u_{R, \beta}$. Since $N y<N u$ on $(R, \infty)$ and also $y(R)=u(R), F_{s}\left(y_{r}(R)\right)=F_{s}\left(u_{r}(R)\right)$ it follows that for $r>R$

$$
\begin{gathered}
F_{s}\left(y_{r}(r)\right)<F_{s}\left(u_{r}(r)\right) \\
y(r)<u(r) .
\end{gathered}
$$

Let $\hat{r}=\hat{r}(r)$ be defined by $y(\hat{r})=u(r)$. By (3.12), clearly $\hat{r}<r$. Since $y_{r r}>0$ and $F_{s s}>0$ we have $F_{s}\left(y_{r}(\hat{r})\right)<F_{s}\left(y_{r}(r)\right)$. Thus,

$$
-\frac{n-1}{r} F_{s}\left(u_{r}(r)\right)<-\frac{n-1}{\hat{r}} F_{s}\left(y_{r}(\hat{r})\right) \text {. }
$$

From this the claim (3.10) is now evident. Combining (3.8), (3.9) and (3.10) we have the basic inequality

$$
-\int_{z(\infty ; R, \beta)}^{-u_{R, \beta}} \frac{n-1}{r} F_{s}\left(z_{r}\right) d z<G\left(u_{R, \beta}\right) .
$$

In order to bring (3.13) to a more transparent form we define

$$
\begin{aligned}
A(R, T) & =-\int_{z(\infty, R, \beta)}^{-T} \frac{n-1}{r} F_{s}\left(z_{r}\right) d z \\
& =(n-1) \beta R^{n-1} \int_{z(\infty ; R, \beta)}^{-T} r(z ; R, \beta)^{-n} d z .
\end{aligned}
$$

We note that since $z(\lambda r ; \lambda R, \beta)=\lambda z(r ; R, \beta)$ for $\lambda>0$, a change of variable in integration in (3.14) yields $A(\lambda R, \lambda T)=A(R, T)$. Thus, $A(R, T)=S(T / R)$ where

$$
S(w)=A(1, w), \quad w>0 .
$$

Inequality (3.13) now becomes

$$
S\left(u_{R, \beta} / R\right)<G\left(u_{R, \beta}\right) .
$$

Since $S_{w}<0$ and $G_{u}>0, T(R ; \beta)$ given by $(3.5)$ is a well-defined $C^{1}$ function of $R$ with the stated properties of the theorem. This completes the proof.

We now establish the asymptotic behavior of $u_{R, \beta}$ as $R \rightarrow 0$ and as $R \rightarrow \infty$. 
THEOREM 3.2. The boundary height $u_{R, \beta}$ as given in Theorem 3.1 is characterized asymptotically by

$$
\begin{aligned}
& u_{R, \beta} \sim \begin{cases}\frac{\beta}{F_{s s}(0)} R \log \frac{a}{R} & n=2 \\
C_{n, \beta} R & n>2 \text { as } R \longrightarrow 0,\end{cases} \\
& u_{R, \beta} \sim u_{\infty, \beta}=G^{-1}(\Phi(0)-\Phi(-\beta)) \text { as } R \longrightarrow \infty \text {, }
\end{aligned}
$$

where $a=\left\{G_{u u}(0) / F_{s s}(0)\right\}^{-1 / 2}, C_{n, \beta}=\int_{1}^{\infty} F_{s}^{-1}\left(\beta r^{-n+1}\right) d r$.

Proof. First we consider the case $R \rightarrow 0$. Using (3.4) we find the asymptotic estimates for $u_{R, \beta}$ from below by determining $T(R ; \beta)$ asymptotically as $R \rightarrow 0$. Equations $(3.5,3.6)$ imply that $T \rightarrow 0$ and

$$
T / R \longrightarrow|z(\infty ; 1, \beta)|=\left\{\begin{array}{ll}
\infty & n=2 \\
C_{n, \beta} & n>2
\end{array} \text { as } R \longrightarrow 0 .\right.
$$

For $n>2$ it follows that

$$
u_{R, \beta} \geqq(1-\varepsilon(R)) C_{n, \beta} R \quad(n>2)
$$

for $0<\varepsilon(R)=o(1)$ as $R \rightarrow 0$.

For $n=2$ we must estimate $S(w)$ from below for large $w$. In the following analysis we write simply $z(r)$ and $r(z)$ for $z(r ; 1, \beta)$ and $r(z ; 1, \beta)$, respectively. Take $\bar{w}>0$ and consider $0>-\bar{w}>$ $-w>z$. We first estimate

$$
\begin{aligned}
-z(r) & \geqq \int_{r(\bar{w})}^{r} F_{s}^{-1}(\beta / \rho) d \rho \\
& \geqq \int_{r(\bar{w})}^{r} \frac{\theta(\bar{w})}{F_{s s}(0)} \cdot \frac{\beta}{\rho} d \rho \\
& =\frac{\beta \theta(\bar{w})}{F_{s s}(0)} \log \frac{r}{r(\bar{w})}
\end{aligned}
$$

where $0<\theta(\bar{w})<1$ and $\theta(\bar{w}) \rightarrow 1$ as $\bar{w} \rightarrow \infty$. Hence,

$$
r(z) \leqq r(\bar{w}) \exp \left\{\frac{F_{s s}(0)}{\beta \theta(\bar{w})}(-z)\right\} .
$$

Thus,

$$
\begin{aligned}
S(w) & =\beta \int_{-\infty}^{-w} r(z)^{-2} d z \\
& \geqq \beta \int_{-\infty}^{-w} r(\bar{w})^{-2} \exp \left\{\frac{-2 F_{s s}(0)}{\beta \theta(\bar{w})}(-z)\right\} d z \\
& =\frac{\beta^{2} \theta(\bar{w})}{2 F_{s s}(0) r(\bar{w})^{2}} \exp \left\{\frac{-2 F_{s s}(0)}{\beta \theta(\bar{w})} w\right\} .
\end{aligned}
$$


Recalling (1.7) we have

$$
0<G(u) \leqq \frac{1}{2} \tau(\bar{u}) G_{u u}(0) u^{2} \quad \text { for } \quad 0<u<\bar{u}
$$

where $\tau(\bar{u}) \geqq 1$ and $\tau(\bar{u}) \rightarrow 1$ as $\bar{u} \rightarrow 0$.

If we take $\delta(\bar{w}, \bar{u})>0$ so that $R<\delta(\bar{w}, \bar{u})$ implies both $T / R>\bar{w}$ and $T<\bar{u}$ we obtain from (3.22), (3.23) and (3.5)

$$
T \geqq \frac{\beta C(\bar{w}, \bar{u})}{r(\bar{w})} \exp \left\{\frac{-F_{s s}(0)}{\beta \theta(\bar{w})} \cdot \frac{T}{R}\right\}
$$

where $C(\bar{w}, \bar{u})=\left[\theta(\bar{w}) / F_{s s}(0) G_{u u}(0) \tau(\bar{u})\right]^{1 / 2}$. We rewrite (3.24) in the form

$$
\frac{T}{R} \geqq \frac{\beta \theta(\bar{w})}{F_{s s}(0)} \log \left\{\frac{\beta C(\bar{w}, \bar{u})}{r(\bar{w})} \cdot \frac{1}{T}\right\} .
$$

Then for any positive function $V(R)$ we have

$$
\frac{T}{R} \geqq \min \left(V, \frac{\beta \theta(\bar{w})}{F_{s s}(0)} \log \left\{\frac{\beta C(\bar{w}, \bar{u})}{r(\bar{w})} \cdot \frac{1}{R V}\right\}\right) .
$$

The best choice for $V(R)$ in the asymptotic estimates must be such that as $R \rightarrow 0$

$$
\begin{aligned}
V & \sim \frac{\beta \theta(\bar{w})}{F_{s s}(0)} \log \left\{\frac{\beta C(\bar{w}, \bar{u})}{r(\bar{w})} \cdot \frac{1}{R V}\right\} \\
& =\frac{\beta \theta(\bar{w})}{F_{s s}(0)} \log \frac{a}{R}+o(V)
\end{aligned}
$$

provided $V \rightarrow \infty$ as $R \rightarrow 0$. On this basis we choose

$$
V(R)=\frac{\beta \theta(\bar{w})}{F_{s s}(0)} \log \frac{a}{R} .
$$

For any $\varepsilon>0$, there exists $\bar{w}(\varepsilon)$ sufficiently large and $\bar{u}(\varepsilon)$ sufficiently small and $\delta(\varepsilon)>0$ such that for $R<\delta(\varepsilon)$ the relations (3.26), (3.27), (3.28) yield

$$
\frac{T}{R} \geqq(1-\varepsilon) \frac{\beta}{F_{s s}(0)} \log \frac{a}{R} .
$$

Thus we have the asymptotic estimate from below

$$
u_{R, \beta} \geqq(1-\varepsilon(R)) \frac{\beta}{F_{s s}(0)} R \log \frac{a}{R} \quad(n=2)
$$

for $0<\varepsilon(R)=o(1)$ as $R \rightarrow 0$.

We now derive asymptotic estimates from above for $u_{R, \beta}$ as 
$R \rightarrow 0$. These estimates stem from the observation that since $N z<$ $N u$ on $r>R$ and $F_{s}\left(z_{r}(R)\right)=F_{s}\left(u_{r}(R)\right)$ hold for $z=z(r ; R, \beta)$ and $u=u(r ; R, \beta)$ we have

$$
\begin{gathered}
F_{s}\left(z_{r}(\hat{R})\right)<F_{s}\left(u_{r}(\hat{R})\right) \\
u_{R, \beta}<|z(\hat{R} ; R, \beta)|+u(\hat{R} ; R, \beta)
\end{gathered}
$$

for any $\hat{R}>R$. We show by an appropriate choice of $\hat{R}$ that $u(\hat{R} ; R, \beta)=o\left(u_{R, \beta}\right)$ as $R \rightarrow 0$, and hence obtain an asymptotic estimate for $u_{R, \beta}$ from the behavior of $|z(\hat{R} ; R, \beta)|$ which is known explicitly.

The estimation of $u(\hat{R} ; R, \beta)$ is achieved by linearizing the equation (1.17) on $(\hat{R}, \infty)$ (for large $\hat{R} / R$ ) and majorizing the solution $u(r)$ by a supersolution $v(r)$ on $(\hat{R}, \infty)$ which is itself a solution of the linearized equation. Suppose $v(r) \in C^{2}[R, \infty)$ satisfies $v>0, v_{r}<0$, $v_{r r}>0, \lim _{r \rightarrow \infty} v=\lim _{r \rightarrow \infty} v_{r}=0$; let $v_{0}=\sup _{r \in(\hat{R}, \infty)} v(r), v_{1}=\sup _{r \in(\hat{R}, \infty)}\left|v_{r}(r)\right|$. Then on $(\hat{R}, \infty)$ there holds

$$
\begin{gathered}
N v<F_{s s}\left(v_{r}\right) v_{r r} \leqq\left(1+\varepsilon_{1}\left(v_{1}\right)\right) F_{s s}(0) v_{r r} \\
G_{u}(v) \geqq\left(1-\varepsilon_{0}\left(v_{0}\right)\right) G_{u u}(0) v
\end{gathered}
$$

where $0<\varepsilon_{0}\left(v_{0}\right)=o(1)$ as $v_{0} \rightarrow 0$ and $0<\varepsilon_{1}\left(v_{1}\right)=o(1)$ as $v_{1} \rightarrow 0$. Thus, the supersolution condition

$$
N v \leqq G_{u}(v) \quad r>\hat{R}
$$

is satisfied if the linear differential inequality

$$
v_{r r} \leqq \frac{1-\varepsilon_{0}\left(v_{0}\right)}{1+\varepsilon_{1}\left(v_{1}\right)} \cdot \frac{G_{u u}(0)}{F_{s s}(0)} v \quad r>\hat{R}
$$

holds. For $0<\varepsilon<1 / 2$ we define

$$
A_{\varepsilon}=\left[(1-\varepsilon) G_{u u}(0) / F_{s s}(0)\right]^{-1 / 2} ;
$$

clearly $A_{\varepsilon} \rightarrow a$ as $\varepsilon \rightarrow 0$. For any $\varepsilon>0$ we can find $\delta(\varepsilon)>0$ so that if $v_{0}+v_{1}<\delta(\varepsilon)$ then $1-\varepsilon<\left(1-\varepsilon_{0}\left(v_{0}\right)\right) /\left(1+\varepsilon_{1}\left(v_{1}\right)\right)$ and hence the function

$$
v(r)=\mathrm{Be}^{-r / A_{\varepsilon}}
$$

satisfies (3.36) for any constant $B$. We now propose to choose $B$ so that the boundary condition

$$
-F_{s}\left(u_{r}(\hat{R})\right)<-F_{s}\left(v_{r}(\hat{R})\right)
$$

holds. By (3.31) it suffices to take $B$ such that $\left|z_{r}(\hat{R})\right| \leqq\left|v_{r}(\hat{R})\right|$. But 


$$
\left|z_{r}(\hat{R})\right|=F_{s}^{-1}\left(\beta(R / \hat{R})^{n-1}\right) \leqq \eta\left(\frac{\hat{R}}{R}\right) \frac{\beta}{F_{s s}(0)}\left(\frac{R}{\hat{R}}\right)^{n-1}
$$

where $\eta(\hat{R} / R)>1$ and $\eta(\hat{R} / R) \rightarrow 1$ as $\hat{R} / R \rightarrow \infty$. Therefore the appropriate choice of $B$ is

$$
B=A_{\varepsilon} \eta\left(\frac{\hat{R}}{R}\right) \frac{\beta}{F_{s s}(0)}\left(\frac{R}{\widehat{R}}\right)^{n-1} e^{\hat{R} / 1_{\varepsilon}} .
$$

Now we have

$$
\begin{aligned}
& v_{1}=\eta\left(\frac{\hat{R}}{R}\right) \frac{\beta}{F_{s s}(0)}\left(\frac{R}{\hat{R}}\right)^{n-1}=0\left(\left(\frac{R}{\hat{R}}\right)^{n-1}\right), \\
& v_{0}=A_{\varepsilon} v_{1}=0\left(\left(\frac{R}{\hat{R}}\right)^{n-1}\right),
\end{aligned}
$$

as $\hat{R} / R \rightarrow \infty \quad\left(A_{\varepsilon}\right.$ is bounded independent of $\left.\varepsilon\right)$. Thus there exists $M(\varepsilon)>0$ such that if $\hat{R} / R>M(\varepsilon)$ then the condition $v_{0}+v_{1}<\delta(\varepsilon)$ is satisfied for $v(r)$ given by (3.37). Consequently, $v(r)$ satisfies (3.35) and (3.38) and hence Theorem 1.1 implies $u(r) \leqq v(r)$ for $r \geqq \hat{R}$. In particular we have the desired estimate

$$
u(\hat{R} ; R, \beta) \leqq \eta\left(\frac{\hat{R}}{R}\right) \frac{\beta A_{\varepsilon}}{F_{s s}(0)}\left(\frac{R}{\hat{R}}\right)^{n-1}
$$

for any $\varepsilon>0$ provided $\hat{R} / R>M(\varepsilon)$. Recalling the properties of $A_{\varepsilon}$ and $\eta(\hat{R} / R)$ we can express this simply as

$$
u(\hat{R} ; R, \beta) \leqq\left(1+\zeta\left(\frac{\hat{R}}{R}\right)\right) \frac{\beta a}{F_{s s}(0)}\left(\frac{R}{\hat{R}}\right)^{n-1}
$$

where $0<\zeta(\hat{R} / R)=o(1)$ as $\hat{R} / R \rightarrow \infty$.

It remains to estimate $|z(\hat{R} ; R, \beta)|$ as $\hat{R} / R \rightarrow \infty$. Writing $\hat{r}=$ $\hat{R} / R$ we recall that $|z(\hat{R} ; R, \beta)|=R|z(\hat{r} ; 1, \beta)|$. We have directly from (3.3) that

$$
|z(\hat{r} ; 1, \beta)| \leqq \begin{cases}(1+\zeta(\hat{r})) \frac{\beta}{F_{s s}(0)} \log \hat{r} & n=2 \\ C_{n, \beta} & n>2\end{cases}
$$

where $0<\zeta(\hat{r})=o(1)$ as $\hat{r} \rightarrow \infty$.

For $n=2$ we combine (3.39) and (3.40) to get

$$
\begin{aligned}
u_{R, \beta} & \leqq R|z(\hat{R} / R ; 1, \beta)|+u(\hat{R} ; R, \beta) \\
& \leqq\left(1+\zeta\left(\frac{\hat{R}}{R}\right)\right) \frac{\beta}{F_{s s}(0)}\left\{R \log \frac{\hat{R}}{R}+\frac{a R}{\hat{R}}\right\} .
\end{aligned}
$$

Taking $\hat{R}=a$ we have 


$$
u_{R, \beta} \leqq(1+\varepsilon(R)) \frac{\beta}{F_{s s}(0)} R \log \frac{a}{R}
$$

where $0<\varepsilon(R)=o(1)$ as $R \rightarrow 0$.

For $n>2$ we may take $\hat{R}=\infty$ and obtain immediately

$$
u_{R, \beta} \leqq C_{n, \beta} R \text {. }
$$

Inequalities (3.30), (3.41) for $n=2$ and (3.19), (3.42) for $n>2$ establish the asymptotic statement (3.17).

Finally we must prove (3.18). Since $T(R ; \beta)$ is bounded above by $u_{\infty, \beta}, G(T)=S(T / R) \rightarrow S(0)$ and thus $T(R ; \beta) \rightarrow G^{-1}(S(0))$ as $R \rightarrow \infty$. But

$$
S(0)=-\int_{z(\infty ; 1, \beta)}^{0} \frac{n-1}{r} F_{s}\left(z_{r}\right) d z=\Phi(0)-\Phi(-\beta)
$$

by (3.9). Thus, $T(R ; \beta) \rightarrow u_{\infty, \beta}$ as $R \rightarrow \infty$. This establishes (3.18) and completes the proof of the theorem.

We conclude this section by specializing the results to the case of two dimensional capillary free surfaces.

THEOREM 3.3. Let $u=u(r ; R, \beta)$ be the solution of

$$
\begin{array}{ll}
\frac{1}{r} \frac{d}{d r}\left(\frac{r u_{r}}{\sqrt{1+u_{r}^{2}}}\right)=\kappa u & r>R \\
-\frac{u_{r}}{\sqrt{1+u_{r}^{2}}}=\beta=\cos \gamma & r=R
\end{array}
$$

for $0 \leqq \gamma<\pi / 2$. Then there holds for $0<R<\infty$

$$
T(R ; \beta)<u_{R, \beta}<a \sqrt{2(1-\sin \gamma)}
$$

where $T(R ; \beta)$ is defined implicitly by

$$
\frac{T}{R \cos \gamma}=\log \left\{\frac{(1-\sin \gamma)}{\cos \gamma} \sqrt{\left.\left(\frac{2 a}{T}\right)^{2}-1\right\}} ;\right.
$$

$a=1 / \sqrt{\kappa}$. Also, $u_{R, \beta}$ is characterized asymptotically by

$$
\begin{aligned}
& u_{R, \beta} \sim \cos \gamma R \log a / R \text { as } R \longrightarrow 0 \\
& u_{R, \beta} \sim a \sqrt{2(1-\sin \gamma)} \text { as } R \longrightarrow \infty .
\end{aligned}
$$

Proof. All of the these results are immediate from Theorems 3.1 and 3.2 with the exception of the implicit relation for $T(R ; \beta)$. To derive the latter we note that

$$
z(r ; 1, \cos \gamma)=\cos \gamma\left\{\cosh ^{-1}(\sec \gamma)-\cosh ^{-1}(r \sec \gamma)\right\}
$$


and hence

$$
\begin{aligned}
S(w) & =\cos \gamma \int_{-\infty}^{-w} r(z ; 1, \cos \gamma)^{-2} d z \\
& =1-\tanh \left(w \sec \gamma+\cosh ^{-1}(\sec \gamma)\right) .
\end{aligned}
$$

Thus, the defining relation (3.5) for $T(R ; \cos \gamma)$ becomes

$$
1-\tanh \left(\frac{T}{R \cos \gamma}+\cosh ^{-1}(\sec \gamma)\right)=\frac{1}{2}\left(\frac{T}{a}\right)^{2} .
$$

From this the implicit relation in the statement of the theorem follows easily.

In the physical case the asymptotic formula for $u_{R, \beta}$ as $R \rightarrow 0$ has been derived formally by Derjaguin [3] using the method of matching asymptotic expansions; the above theorem provides a rigorous justification of this result. The lower bound $T(R ; \beta)$, valid for all $0<R<\infty$ and asymptotically exact as $R$ tends to zero or infinity, seems to be new even in the physical case. The upper bound is the same as that of Johnson and Perko [9].

4. Behavior near the boundary. The following lemma gives a majorant and minorant for the solution $u(r ; R, \beta)$ on an interval $(R, \hat{R})$ and an estimate for the error over that interval as $R \rightarrow 0$. In the subsequent theorem we formulate in dimensionless quantities certain asymptotic estimates for $u(r ; R, \beta)$ in a neighborhood of the boundary.

Lemma 4.1. Let $u=u(r ; R, \beta)$ be as in Theorem 2.1 and let $z=z(r ; R, \beta)$ be given by (3.3), $0<\beta \leqq 1$. The functions

$$
\begin{gathered}
w=w(r ; R, \beta)=u(R ; R, \beta)+z(r ; R, \beta) \\
v=v(r ; R, \beta)=u(\hat{R} ; R, \beta)-z(\hat{R} ; R, \beta)+z(r ; R, \beta),
\end{gathered}
$$

where $\hat{R}=a$ if $n=2, \hat{R}=\infty$ if $n>2$, satisfy

$$
w(r ; R, \beta)<u(r ; R, \beta)<v(r ; R, \beta)
$$

on $R<r<\hat{R}$. Furthermore, as $R \rightarrow 0$

$$
\begin{aligned}
\sup _{r \in(R \hat{R})}\{v(r ; R, \beta)-u(r ; R, \beta)\} & =\sup _{r \in(R, \hat{R})}\{u(r ; R, \beta)-w(r ; R, \beta)\} \\
& =o(u(R ; R, \beta)) .
\end{aligned}
$$

Proof. Since $N u>0$ while $N w=N v=0$ on $R<r<\hat{R}$ and $w_{r}(R)=v_{r}(R)=u_{r}(R), w(R)=u(R), v(\hat{R})=u(\hat{R})$, inequality (4.3) is evident. Also, 


$$
\begin{aligned}
\sup _{r \in(R, \hat{R})}\{v(r)-u(r)\} & =v(R)-u(R) \\
& =u(\hat{R})+|z(\hat{R})|-u(R) \\
& =u(\hat{R})-w(\hat{R}) \\
& =\sup _{r \in(R, \hat{R})}\{u(r)-w(r)\} .
\end{aligned}
$$

We need only show that

$$
0<u(\hat{R} ; R, \beta)+|z(\hat{R} ; R, \beta)|-u(R ; R, \beta)=o(u(R ; R, \beta))
$$

as $R \rightarrow 0$. But this follows from the proof of Theorem 3.2 -in particular, from (3.39), (3.40), (3.19) and (3.30).

THEOREM 4.2. For $0<\theta<1$, let $1<\lambda_{\theta}(R)<\infty$ be defined by $u\left(\lambda_{\theta}(R) R ; R, \beta\right)=\theta u(R ; R, \beta)$. Then for any $\varepsilon>0$ there exists $\delta(\varepsilon)>0$ such that if $R<\delta(\varepsilon)$ then

$$
\begin{aligned}
& \left(\frac{R}{a}\right)^{\theta+\varepsilon-1}<\lambda_{\theta}(R)<\left(\frac{R}{a}\right)^{\theta-\varepsilon-1} \quad n=2 \\
& \rho_{n, \beta}(\theta+\varepsilon)<\lambda_{\theta}(R)<\rho_{n, \beta}(\theta-\varepsilon) \quad n>2
\end{aligned}
$$

where $\rho_{n, \beta}(t)$ is a decreasing, continuous function on $0<t<1$ with $\lim _{t \rightarrow 0} \rho_{n, \beta}(t)=\infty, \lim _{t \rightarrow 1} \rho_{n, \beta}(t)=1$.

Proof. Let $\lambda_{\theta}^{\prime}(R)$ and $\lambda_{\theta}^{\prime \prime}(R)$ be defined by $w\left(\lambda_{\theta}^{\prime}(R) R ; R, \beta\right)=$ $v\left(\lambda_{\theta}^{\prime \prime}(R) R ; R, \beta\right)=\theta u(R ; R, \beta)$. Then $\lambda_{\theta}^{\prime}(R)<\lambda_{\theta}(R)<\lambda_{\theta}^{\prime \prime}(R)$ by (4.3). Also from Lemma 4.1 we conclude

$$
\begin{aligned}
& \left|z\left(\lambda_{\theta}^{\prime}(R) R ; R, \beta\right)\right|=(1-\theta) u(R ; R, \beta) \\
& \left|z\left(\lambda_{0}^{\prime \prime}(R) R ; R, \beta\right)\right| \leqq(1-\theta+\varepsilon) u(R ; R, \beta)
\end{aligned}
$$

where $0<\varepsilon=o(1)$ as $R \rightarrow 0$. For $n=2, u(R ; R, \beta) / R \rightarrow \infty$ and hence $\lambda_{\theta}^{\prime}(R), \lambda_{0}^{\prime \prime}(R) \rightarrow \infty$ as $R \rightarrow 0$. We recall that

$$
|z(\rho R ; R, \beta)=R| z(\rho ; 1, \beta) \mid \sim R \frac{\beta}{F_{s s}(0)} \log \rho \text { as } \rho \longrightarrow \infty .
$$

Now invoking Theorem 3.2 we conclude from (4.7) that

$$
\begin{aligned}
& \log \lambda_{\rho}^{\prime}(R) \geqq(1-\theta-\varepsilon) \log \frac{a}{R} \\
& \log \lambda_{\rho}^{\prime \prime}(R) \leqq(1-\theta+\varepsilon) \log \frac{a}{R}
\end{aligned}
$$

where, again, $0<\varepsilon=o(1)$. This proves (4.5). For $n>2$ we construct $\rho_{n, \beta}(t)$ by solving the equation 


$$
\left|z\left(\rho_{n, \beta}(t) ; 1, \beta\right)\right|=(1-t)|z(\infty ; 1, \beta)|=(1-t) C_{n, \beta} .
$$

Clearly $\rho_{n, \beta}(t)$ is well-defined for $0<t<1$ and possesses the properties stated in the theorem. Now (4.7) and Theorem 3.2 imply

$$
\begin{aligned}
& \left|z\left(\lambda_{\rho}^{\prime}(R) ; 1, \beta\right)\right| \geqq(1-\theta-\varepsilon) C_{n, \beta}=\left|z\left(\rho_{n, \beta}(\theta+\varepsilon) ; 1, \beta\right)\right| \\
& \left|z\left(\lambda_{\rho}^{\prime \prime}(R) ; 1, \beta\right)\right| \leqq(1-\theta+\varepsilon) C_{n, \beta}-\left|z\left(\rho_{n, \beta}(\theta-\varepsilon) ; 1, \beta\right)\right|
\end{aligned}
$$

from which (4.6) follows.

We note that the asymptotic characterization of $\lambda_{\theta}(R)$ given in the above theorem depends crucially upon the asymptotic exactness of the boundary height estimates of Theorem 3.2.

5. Extensions to other equations. The results of the preceding sections may be extended to a more general class of variational equations. Let $A(p) \in C^{2}\left(\boldsymbol{R}^{n}\right)$ and $B(x, u) \in C^{2}(\bar{\Omega} \times \boldsymbol{R})$. We consider the boundary value problem

$$
\begin{array}{ll}
\frac{\partial}{\partial x_{i}} A_{p_{i}}(\nabla u)=B_{u}(x, u) & x \in \Omega \\
\nu_{i} A_{p_{i}}(\nabla u)=\varphi_{u}(x, u) & x \in \Sigma
\end{array}
$$

where $\varphi(x, u)$ is prescribed data as before. These equations arise from a variational functional with an integrand separated in the $(x, u)$ and $p$ variables; namely,

$$
J[u]=\int_{\Omega}\{A(\nabla u)+B(x, u)\} d x-\int_{\Sigma} \varphi(x, u) d S .
$$

We assume

$$
A_{p_{\imath}}(0)=0 \text { and } B_{u}(x, 0)=0 \text { for all } x \in \bar{\Omega},
$$

so that $u \equiv 0$ is a solution of (5.1). Thus it is natural to require the condition at infinity (1.5). We also assume the convexity conditions

$$
\begin{aligned}
& A_{p_{i} p_{j}}(p) \xi_{i} \xi_{j}>0 \text { for all } p \in \boldsymbol{R}^{n}, 0 \neq \xi \in \boldsymbol{R}^{n}, \\
& B_{u u}(x, u)>0 \text { for all }(x, u) \in \bar{\Omega} \times \boldsymbol{R} .
\end{aligned}
$$

Under these conditions a maximum principle analogous to Theorem 1.1 holds for solutions of $(5.1,5.2)$.

We must, in addition to (5.5), impose a structure condition on the equation (5.1) similar to the conditions of $\S 2$ for symmetric variational equations. Let $F(s)$ be given satisfying the conditions of $\S 2$. We require that

$$
F_{p_{i} p_{j}}(p) \xi_{\imath} \xi_{j} \leqq A_{p_{i} p_{j}}(p) \xi_{i} \xi_{j} \leqq \mu F_{p_{i} p_{j}}(p) \xi_{i} \xi_{j}
$$


for all $p \in \boldsymbol{R}^{n}, \xi \in \boldsymbol{R}^{n}$, where $\mu \geqq 1$ is a constant independent of $p$ and $\xi$. In the special case when $F(s)=\sqrt{1+s^{2}}$ the equation (5.1) is said to be of mean curvature type.

For the symmetric variational problem $(1.8,1.9)$ a rotationally symmetric solution in $\widetilde{B}_{R}$ is a solution of the ordinary differential equations $(1.17,1.18)$. We now propose to find analogous ordinary differential equations whose solutions yield rotationally symmetric sub- and super-solutions of problem $(5.1,5.2)$.

Let $v \in C^{2}[R, \infty)$ and consider $v$ as a function of $x=\left(x_{1}, \cdots, x_{n}\right)$ by $v(x)=v(r), r=|x|$. Then

$$
\begin{aligned}
v_{x_{i}} & =v_{r} \frac{x_{i}}{r} \\
v_{x_{i} x_{j}} & =v_{r r} \frac{x_{i} x_{j}}{r^{2}}+\frac{1}{r} v_{r}\left\{\delta_{i j}-\frac{x_{i} x_{j}}{r^{2}}\right\} \\
& =v_{r r} \frac{v_{x_{i}} v_{x_{j}}}{v_{r}^{2}}+\frac{1}{r} v_{r}\left\{\delta_{i j}-\frac{v_{x_{i}} v_{x_{j}}}{v_{r}^{2}}\right\} .
\end{aligned}
$$

Also, with $s=|p|$,

$$
F_{p_{i} p_{j}}(p)=F_{s s}(s) \frac{p_{i} p_{j}}{s^{2}}+\frac{1}{s} F_{s}(s)\left\{\delta_{i j}-\frac{p_{i} p_{j}}{s^{2}}\right\} .
$$

From this it follows that

$$
\begin{aligned}
F_{p_{i} p_{j}}(p) \xi_{i} \xi_{j} & =F_{s s}(s) \frac{(p \cdot \xi)^{2}}{s^{2}}+\frac{1}{s} F_{s}(s)\left\{|\xi|^{2}-\frac{(p \cdot \xi)^{2}}{s^{2}}\right\} \\
& =F_{s s}(s)\left|\xi^{\prime}\right|^{2}+\frac{1}{s} F_{s}(s)\left|\xi^{\prime \prime}\right|^{2}
\end{aligned}
$$

where $\xi^{\prime}$ and $\xi^{\prime \prime}$ are the projections of $\xi$ in the directions parallel and perpendicular to $p$, respectively.

Applying the operator on the left hand side of (5.1) to $v$ we get, using (5.8),

$$
\begin{aligned}
\frac{\partial}{\partial x_{i}} A_{p_{i}}(\nabla v) & =A_{p_{i} p_{j}}(\nabla v) v_{x_{i} x_{j}} \\
& =E(\nabla v) v_{r r}+[T(\nabla v)-E(\nabla v)] \frac{1}{r} v_{r}
\end{aligned}
$$

where

$$
E(p)=A_{p_{i} p_{i}}(p) p_{i} p_{j} / s^{2}, T(p)=A_{p_{i} p_{i}}(p) .
$$

Taking $\xi=p$ in (5.10) it is evident that (5.7) implies

$$
F_{s s}(s) \leqq E(p) \leqq \mu F_{s s}(s), \quad s=|p| .
$$


We can estimate $T(p)-E(p)$ as follows. Let $\xi^{(1)}, \cdots, \xi^{(n)}$ be an orthonormal basis for $\boldsymbol{R}^{n}$ with $\xi^{(1)}=p / s$. Then

$$
\begin{aligned}
T(p) & =\sum_{k=1}^{n} A_{p_{i} p_{j}}(p) \xi_{i}^{(k)} \xi_{j}^{(k)} \\
& =E(p)+\sum_{k=2}^{n} A_{p_{i} p_{j}}(p) \xi_{i}^{(k)} \xi_{j}^{(k)} \cdot{ }^{2}
\end{aligned}
$$

By (5.7) and (5.10), for $k=2, \cdots, n$, we have

$$
\frac{1}{s} F_{s}(s) \leqq A_{p_{i} p_{j}} \xi_{i}^{(k)} \xi_{j}^{(k)} \leqq \mu \frac{1}{s} F_{s}(s) .
$$

Consequently

(5.14) $\quad \frac{(n-1)}{s} F_{s}(s) \leqq T(p)-E(p) \leqq \frac{(n-1) \mu}{s} F_{s}(s), \quad s=|p|$

In addition to (5.6) let us impose

$$
B_{u}(x, u) \leqq G_{u}(u) \text { for all }(x, u) \in \bar{\Omega} \times \boldsymbol{R}
$$

for some given $G(u)$ as in $\S 2$. Suppose now that we seek a sub-solution $v(r)$ of $(5.6,5.2)$ on $\widetilde{B}_{R}$ with the properties $v_{r}<0, v_{r r}>0$ for $r>R$. By the above discussion

$$
\begin{aligned}
\frac{\partial}{\partial x_{i}} A_{p_{i}}(\nabla v) & =E(\nabla v) v_{r r}+[T(\nabla v)-E(\nabla v)] \frac{1}{r} v_{r} \\
& \geqq F_{s s}\left(v_{r}\right) v_{r r}+\frac{(n-1) \mu}{r} F_{s}\left(v_{r}\right) \\
& =r^{-\alpha} \frac{d}{d r}\left(r^{\alpha} F_{s}\left(v_{r}\right)\right)
\end{aligned}
$$

where $\alpha=(n-1) \mu$. Hence, if $v(r)$ is a solution of

$$
N_{\alpha} v=r^{-\alpha} \frac{d}{d r}\left(r^{\alpha} F_{s}\left(v_{r}\right)\right)=G_{u}(v) \quad r>R
$$

then $v$ satisfies the sub-solution condition

$$
\frac{\partial}{\partial x_{i}} A_{p_{i}}(\nabla v) \geqq B_{u}(x, v) \quad \text { on } \quad \widetilde{B}_{R} .
$$

We must now deal with the boundary condition. Since

$$
\nu_{i}=-\frac{x_{i}}{r}=-\frac{v_{x_{i}}}{v_{r}}=\frac{v_{x_{i}}}{|\nabla v|},
$$

we have

${ }^{2}$ We retain the summation convention on indices $i$ and $j$. 


$$
\nu_{i} A_{p_{i}}(\nabla v)=\frac{v_{x_{i}}}{|\nabla v|} A_{p_{i}}(\nabla v) \quad \text { on } \quad \partial \widetilde{B}_{R}
$$

Recalling (5.4) we see that

$$
\begin{aligned}
\frac{p_{i}}{|p|} A_{p_{i}}(p) & =\frac{p_{i} p_{j}}{|p|} \int_{0}^{1} A_{p_{i} p_{j}}(t p) d t \\
& =|p| \int_{0}^{1} E(t p) d t \\
& \leqq \mu|p| \int_{0}^{1} F_{s s}(t s) d t \\
& =-\mu F_{s}(s), \text { if } s=-|p| .
\end{aligned}
$$

With $p=\nabla v$ and $s=v_{r}$ this yields

$$
\nu_{i} A_{p_{i}}(\nabla v) \leqq-\mu F_{s}\left(v_{r}\right) \text { on } \partial \widetilde{B}_{R} .
$$

Suppose there exists a constant $0<\beta \leqq 1 / \mu$ such that $\varphi_{u}(x, u) \geqq \mu \beta$ for all $(x, u) \in \Sigma \times \boldsymbol{R}$. Now if $v(r)$ satisfies

$$
-F_{s}\left(v_{r}\right)=\beta \quad r=R
$$

then $v$ satisfies the sub-solution boundary condition

$$
\nu_{i} A_{p_{i}}(\nabla v) \leqq \varphi_{u}(x, v) \quad \text { on } \quad \partial \widetilde{B}_{R} \text {. }
$$

In conclusion, a solution of $(5.16,5.18)$ is a sub-solution of $(5.1,5.2)$. Super-solutions are dealt with in an analogous manner. We state these results in the following theorem.

THEOREM 5.1. Suppose that (5.7) holds where $F(s)$ is given satisfying $(2.3,2.5)$ and that

(i) $\mu G_{u}^{(2)}(u) \leqq B_{u}(x, u) \leqq G_{u}^{(1)}(u)$ for all $(x, u) \in \widetilde{B}_{R} \times \boldsymbol{R}$,

(ii) $\mu \beta_{1} \leqq \varphi_{u}(x, u) \leqq \beta_{2}$ for all $(x, u) \in \partial \widetilde{B}_{R} \times \boldsymbol{R}$, where $G^{(k)}(u)$ are given satisfying (1.7) and $\beta_{k}$ are constants with $0<\mu \beta_{1}, \beta_{2} \leqq 1$. Let $v^{(k)}(r)(k=1,2)$ be solutions of (cf. 5.16)

$$
\begin{array}{ll}
N_{\alpha_{k}} v^{(k)}=G_{u}^{(k)}\left(v^{(k)}\right) & r>R \\
-F_{s}\left(v_{r}^{(k)}\right)=\beta_{k} & r=R \\
\lim _{r \rightarrow \infty} v^{(k)}=0, &
\end{array}
$$

with $\alpha_{1}=(n-1) \mu, \alpha_{2}=(n-1) / \mu$. Suppose also that $v_{r}^{(k)}<0$ and $v_{r r}^{(k)}>0$ on $r>R$. Then $v^{(1)}$ and $v^{(2)}$ are sub-and super-solutions, respectively, of the boundary value problem $(5.1,5.2)$ on $\widetilde{B}_{R}$ and hence for the solution $u(x)$ of the problem there holds $v^{(1)} \leqq u \leqq v^{(2)}$ on $\widetilde{B}_{R}$. 
A corresponding theorem holds when $\beta^{(k)}<0$, in which case $v_{r}^{(k)}>0$ and $v_{r r}^{(k)}<0$. So we can treat the case when $\varphi_{u}(x, u)$ is of either sign or when it changes sign.

Theorem 2.1 extends immediately to yield the existence of a unique solution $v=v(r ; R, \beta, \alpha)$ of $(5.16,5.18)$ for any $0<R<\infty$, $0 \leqq \beta \leqq 1,0<\alpha<\infty$; the properties stated in Theorem 2.1 continue to hold for $v(r ; R, \beta, \alpha)$.

The asymptotic estimates of Theorem 3.2 admit the following generalization to $v_{R, \beta, \alpha}=v(R ; R, \beta, \alpha)$.

THEOREM 5.2. For $\varepsilon(R)=o(1)$ as $R \rightarrow 0$, there holds

$$
\begin{aligned}
(1-\varepsilon(R)) \frac{\beta a}{F_{s s}(0)}\left[\frac{R}{(1-\alpha) a}\right]^{\alpha} & \leqq v_{R, \beta, \alpha} \leqq(1+\varepsilon(R)) \frac{2 \beta a}{F_{s s}(0)}\left[\frac{R}{(1-\alpha) a}\right]^{\alpha} \\
\text { for } \quad 0<\alpha<1, & 0<1, \\
v_{R, \beta, 1} & =(1+\varepsilon(R)) \frac{\beta}{F_{s s}(0)} R \log \frac{a}{R}, \\
v_{R, \beta, \alpha} & =(1+\varepsilon(R)) C_{\beta, \alpha} R \text { for } 1<\alpha<\infty,
\end{aligned}
$$

where $C_{\beta, \alpha}=\int_{1}^{\infty} F_{s}^{-1}\left(\beta r^{-\alpha}\right) d r, a=\left[G_{u u}(0) / F_{s s}(0)\right]^{-1 / 2} . \quad$ Also, $v_{R, \beta, \alpha} \sim$ $G^{-1}(\Phi(0)-\Phi(-\beta))$ as $R \rightarrow \infty$ for $0<\alpha<\infty$.

The details of proof appear in Turkington [11].

6. Acknowledgment. The author is deeply indebted to his dissertation advisor Professor Robert Finn under whose guidance this work was completed.

APPENDIX. The construction of sub- and super-solutions carried out in Theorem 5.1 follows the work of Jenkins [7], [8] concerning a generalization of the class of equations of minimal surface type. In this appendix we use the techniques discussed in $\S 5$ to give theorems which contain some of the results of Jenkins.

We consider a general homogeneous quasi-linear elliptic equation

$$
Q u=a_{i j}(x, \nabla u) u_{x_{i} x_{j}}=0
$$

on a domain (specified later) in $\boldsymbol{R}^{n}$. We assume that $a_{i j}(x, p) \epsilon$ $C^{0}\left(\boldsymbol{R}^{n} \times \boldsymbol{R}^{n}\right)$ and $a_{i j}(x, p)=a_{j i}(x, p)$ for all $i, j=1, \cdots, n$. The requirement on $Q u$ which is basic to our considerations is the structure condition:

$$
F_{p_{i} p_{j}}(p) \xi_{i} \xi_{j} \leqq a_{i j}(x, p) \xi_{i} \xi_{j} \leqq \mu(|p|) F_{p_{i} p_{j}}(p) \xi_{i} \xi_{j}
$$

for all $x \in \boldsymbol{R}^{n}, p \in \boldsymbol{R}^{n}, \xi \in \boldsymbol{R}^{n}$, where $\mu(s) \in C^{0}[0, \infty)$ with $\mu(s) \geqq 1$. As 
in $\S 5, F(s)$ is an even $C^{2}$ function satisfying (1.6), (2.3), (2.5). We note that (A. 2) is more general than its counterpart in $\$ 5$ since $\mu(s)$ may be unbounded as $s \rightarrow \infty$. We intend to find growth conditions on $\mu(s)$ as $s \rightarrow \infty$ which allow the construction of rotationally symmetric super-solutions of (A. 1) on $\widetilde{B}_{R}$.

We seek a function $v(r) \in C^{2}(R, \infty), r=|x|$, such that

$$
\begin{gathered}
Q v \leqq 0 \quad|x|>R, \\
v_{r}<0, v_{r r}>0 \quad r>R, \\
\lim _{r \rightarrow R} v_{r}(r)=-\infty .
\end{gathered}
$$

Since any such $v(r)$ is determined only up to an additive constant we require that $v\left(R_{0}\right)=0$ for some fixed $R_{0}>R$.

THEOREM A.1. A function $v=v\left(r ; R, R_{0}\right)$ satisfying (A. 3, A. 4, A. 5) exists provided

(A. 6)

$$
\int_{1}^{\infty} F_{s s}(s) \mu(s) d s<\infty
$$

Furthermore, $\lim _{r \rightarrow R} v\left(r ; R, R_{0}\right)$ is finite provided

$$
\int_{1}^{\infty} s F_{s s}(s) \mu(s) d s<\infty .
$$

Proof. Following the reasoning of Theorem 5.1 we have that if $v(r)$ satisfies (A. 4) then

(A. 8)

$$
Q v=a_{i j}(x, \nabla v) \frac{v_{x_{i}} v_{x_{j}}}{v_{r}^{2}} v_{r r}+\left\{a_{i i}(x, \nabla v)-a_{i j}(x, \nabla v) \frac{v_{x_{i}} v_{x_{j}}}{v_{r}^{2}}\right\} \frac{1}{r} v_{r}
$$

$$
\leqq \mu\left(v_{r}\right) F_{s s}\left(v_{r}\right) v_{r r}+\frac{(n-1)}{r} F_{s}\left(v_{r}\right)
$$

(where we extend $\mu(s)$ as an even function on $(-\infty, \infty)$ for convenience). Hence, the super-solution condition (A. 3) is implied by

$$
\text { (A. 9) } \quad \mu\left(v_{r}\right) F_{s s}\left(v_{r}\right) v_{r r}=-\frac{(n-1)}{r} F_{s}\left(v_{r}\right) \quad R<r<\infty \text {. }
$$

Introducing $s(r)=v_{r}(r)$, we have $s<0, s_{r}>0$ and so we may consider the inverse function $r(s)$. In terms of the latter, (A. 9) becomes

$$
\frac{\mu(s) F_{s s}(s)}{(n-1) F_{s}(s)}=\frac{r_{s}}{r} \quad-\infty<s<0 .
$$

Evidently, the required function $v(r)$ is given by 


$$
v\left(r ; R, R_{0}\right)=\int_{R_{0}}^{r} \rho_{R}^{-1}\left(r^{\prime}\right) d r^{\prime}
$$

where

$$
\rho_{R}(s)=R \exp \left\{-\int_{-\infty}^{s} \frac{\mu\left(s^{\prime}\right) F_{s s}\left(s^{\prime}\right)}{(n-1) F_{s}\left(s^{\prime}\right)} d s^{\prime}\right\}
$$

Recalling that $F_{s}(s) \sim-1$ as $s \rightarrow-\infty$ we see that the convergence of the above integral is equivalent to hypothesis (A.6). Clearly, $\rho_{R}(s)$ is a strictly increasing $C^{1}$ function on $-\infty<s<0$. Also, (A. 5) is satisfied since $\rho_{R}(-\infty)=R$. Finally, to verify the finiteness of $v\left(R ; R, R_{0}\right)$ we compute

$$
\begin{aligned}
v\left(R ; R, R_{0}\right) & =-\int_{R}^{R_{0}} \rho_{R}^{-1}(r) d r \\
& =-\int_{-\infty}^{s\left(R_{0}\right)} s \frac{d \rho_{R}}{d s} d s \\
& =\int_{-\infty}^{s\left(R_{0}\right)} \frac{s \rho_{R}(s) \mu(s) F_{s s}(s)}{(n-1) F_{s}(s)} d s<\infty
\end{aligned}
$$

by hypothesis (A. 7), since $\rho_{R}(s) \sim R$ and $F_{s}(s) \sim-1$ as $s \rightarrow-\infty$.

The construction of a sub-solution can be carried out in an analogous manner.

When $\mu \equiv 1$ in the above theorem, $Q u=\left(\partial / \partial x_{i}\right) F_{p_{i}}(\nabla u)$. In this case, we have $v\left(r ; R, R_{0}\right)=z(r ; R, 1)+\left|z\left(R_{0} ; R, 1\right)\right|$ and conditions (2.3) and (2.5), respectively, imposed on the Legendre transform of $F(s)$.

When $F(s)=W(s)=\sqrt{1+s^{2}}$, Theorem A. 1 yields a result of Jenkins [7] (where $n=2$ ). In this paper the author defines

$$
\begin{aligned}
2 E(x, p) & =\frac{\left(1+p_{1}^{2}\right) a_{11}+2 p_{1} p_{2} a_{12}+\left(1+p_{2}^{2}\right) a_{22}}{\sqrt{1+p_{1}^{2}+p_{2}^{2}} \sqrt{\overline{a_{11} a_{22}-a_{12}^{2}}}} \\
E^{*}(s) & =\sup _{x \in R^{2},|p|=s} E(x, p) .
\end{aligned}
$$

It follows by Lemma 2 of [7] that

$$
2 E=\sqrt{\alpha_{2} / \alpha_{1}}+\sqrt{\alpha_{1} / \alpha_{2}}
$$

where

$$
\alpha_{1}(x, p)=\inf _{\xi \neq 0} \frac{a_{i j}(x, p) \xi_{i} \xi_{j}}{W_{p_{i} p_{j}}(p) \xi_{i} \xi_{j}}, \quad \alpha_{2}(x, p)=\sup _{\xi \neq 0} \frac{a_{i j}(x, p) \xi_{i} \xi_{j}}{W_{p_{i} p_{j}}(p) \xi_{i} \xi_{j}}
$$

By replacing $Q u$ by an equivalent operator we may assume $\alpha_{1} \equiv 1$. Thus, in (A. 2) we now may take 


$$
\mu(s) \sup _{x \in R^{2},|p|=8} \alpha_{2}(x, p)
$$

Recalling $W_{s s}(s) \sim s^{-3}$ as $s \rightarrow \infty$ we see that the conditions (A. 6) and (A. 7) are equivalent the following conditions given in [7], respectively:

$$
\int_{1}^{\infty} \frac{E^{*}(s)^{2}}{s^{3}} d s<\infty, \int_{1}^{\infty} \frac{E^{*}(s)^{2}}{s^{2}} d s<\infty
$$

\section{REFERENCES}

1. P. Concus and R. Finn, On capillary free surfaces in the absence of gravity, Acta. Math., 132 (1974), 177-198.

2. - On capillary free surfaces in a gravitational field, Acta. Math., 132 (1974), 207-223.

3. B. Derjaguin, Theory of the distortion of a plane surface of a liquid by small objects and its application to the measurement of the contact angle of thin filaments and fibers, Dokl. Akad. Nauk. USSR (51) (1942), 519-522.

4. R. Finn, Some comparison properties and bounds for capillary surfaces, Anniversary Volume for I. N. Vekua, Moscow Math. Soc.

5. C. Gerhardt, On the exterior capillary problem, Math. Zeit., 136 (1974), 345-349.

6. - A note on the exterior capillary problem, Math. Zeit., 142 (1975), 203-204.

7. H. B. Jenkins, Super-solutions for quasi-linear elliptic equations, Arch. Rat. Mech. Anal., 16 (1964), 402-410.

8. On the behavior of solutions of quasi-linear elliptic equations, Trans. Amer. Math. Soc., 19 (1965), 407-516.

9. W. E. Johnson and L. M. Perko, Interior and exterior boundary value problems from the theory of the capillary tube, Arch. Rat. Mech. Anal., 29 (1968),125-143.

10. L. M. Perko, A class of superquadratic boundary value problems of capillary type with application to singularly perturbed problems, to appear in Arch. Rat. Mech. Anal., (1979).

11. B. Turkington, Stanford Dissertation, May 1978.

Received March 28, 1979.

NORTHWESTERN UNIVERSITY

Evanston, IL 60201 


\section{PACIFIC JOURNAL OF MATHEMATICS}

\section{EDITORS}

DONALD BABBITT (Managing Editor)

University of California

Los Angeles, CA 90024

Hugo Rossi

University of Utah

Salt Lake City, UT 84112

C. C. MOORE and ANDREW OGG

University of California

Berkeley, CA 94720
J. DUGUNDJI

Department of Mathematics

University of Southern California

Los Angeles, CA 90007

R. FinN and J. Milgram

Stanford University

Stanford, CA 94305

\section{ASSOCIATE EDITORS}
E. F. BECKENBACH
B. H. NeumanN
F. WOLF
K. YoSHIDA

\section{SUPPORTING INSTITUTIONS}

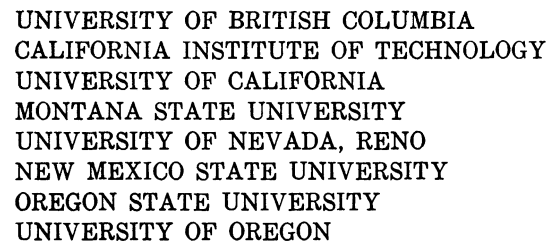

UNIVERSITY OF BRITISH COLUMBIA CALIFORNIA INSTITUTE OF TECHNOLOGY UNIVERSITY OF CALIFORNIA MONTANA STATE UNIVERSITY UNIVERSITY OF NEVADA, RENO NEW MEXICO STATE UNIVERSITY OREGON STATE UNIVERSITY UNIVERSITY OF OREGON

\author{
UNIVERSITY OF SOUTHERN CALIFORNIA \\ STANFORD UNIVERSITY \\ UNIVERSITY OF HAWAII \\ UNIVERSITY OF TOKYO \\ UNIVERSITY OF UTAH \\ WASHINGTON STATE UNIVERSITY \\ UNIVERSITY OF WASHINGTON
}

The Supporting Institutions listed above contribute to the cost of publication of this Journal, but they are not owners or publishers and have no responsibility for its content or policies.

Mathematical papers intended for publication in the Pacific Journal of Mathematics should be in typed form or offset-reproduced, (not dittoed), double spaced with large margins. Please do not use built up fractions in the text of the manuscript. However, you may use them in the displayed equations. Underline Greek letters in red, German in green, and script in blue. The first paragraph or two must be capable of being used separately as a synopsis of the entire paper. Please propose a heading for the odd numbered pages of less than 35 characters. Manuscripts, in triplicate, may be sent to any one of the editors. Please classify according to the scheme of Math. Reviews, Index to Vol. 39. Supply name and address of author to whom proofs should be sent. All other communications should be addressed to the managing editor, or Elaine Barth, University of California, Los Angeles, California, 90024.

50 reprints to each author are provided free for each article, only if page charges have been substantially paid. Additional copies may be obtained at cost in multiples of 50 .

The Pacific Journal of Mathematics is issued monthly as of January 1966. Regular subscription rate: $\$ 84.00$ a year (6 Vols., 12 issues). Special rato: $\$ 42.00$ a year to individual members of supporting institutions.

Subscriptions, orders for numbers issued in the last three calendar years, and changes of address shoud be sent to Pacific Journal of Mathematics, P.O. Box 969, Carmel Valley, CA 93924, U.S.A Old back numbers obtainable from Kraus Periodicals Co., Route 100, Millwood, NY 10546.

PUBLISHED BY PACIFIC JOURNAL OF MATHEMATICS, A NON-PROFIT CORPORATION

Printed at Kokusai Bunken Insatsusha (International Academic Printing Co., Ltd.). 8-8, 3-chome, Takadanobaba, Shinjuku-ku, Tokyo 160, Japan. 


\section{Pacific Journal of Mathematics \\ Vol. 88, No. $2 \quad$ April, 1980}

Reinhold Böhme, Stefan Hildebrandt and Engelbert Tausch, The two-dimensional analogue of the catenary ................. 247

Jean Ellen Taylor, Nonexistence of F-minimizing embedded disks ........ 279

Claus Gerhardt, A free boundary value problem for capillary surfaces ..... 285

Enrico Giusti, Generalized solutions for the mean curvature equation . . . . . 297

Jin-Tzu Chen, On the existence of capillary free surfaces in the absence of gravity.................................... 323

Leon M. Simon, Regularity of capillary surfaces over domains with corners.......................................... 363

Nicholas Jacob Korevaar, On the behavior of a capillary surface at a re-entrant corner.................................... 379

Henry Wente, The symmetry of sessile and pendent drops ............ 387

E. Gonzalez, Umberto Massari and I. Tamanini, Existence and regularity for the problem of a pendent liquid drop ...................... 399

Henry Wente, The stability of the axially symmetric pendent drop ........ 421

David Siegel, Height estimates for capillary surfaces . . . . . . . . . . . . 471

Bruce Edward Turkington, Height estimates for exterior problems of capillarity type ................................ 517

Robert Finn, The sessile liquid drop. I. Symmetric case .............. 541 